\title{
Synthesis and Evaluation of Antihyperglycemic Activity of 1,4-Benzothiazepine-2-One Derivatives in Alloxan Induced Diabetic Rats
}

V. K. SRIVASTAV AND MEENA TIWARI*

Department of Pharmacy, Laboratory of Medicinal and Pharmaceutical Chemistry, Shri Govindram Seksaria Institute of Technology and Science, Indore, Madhya Pradesh 452003, India

Srivastav et al.: Synthesis and Antihyperglycemic Activity of 1,4-Benzothiazepine-2-One Derivatives

\begin{abstract}
The present study reported the synthesis and antihyperglycemic activity of 1,4-benzothiazepines (1,4-benzothiazepine-2-one derivatives) in alloxan-induced diabetic rats. 1,4-benzothiazepines (3a-i) were synthesized via Friedel-Crafts acylation, reduction and microwave assisted $\mathrm{S}$-alkylation reactions and characterized by Fourier transform infrared spectroscopy, proton nuclear magnetic resonance, mass and elemental analysis. In silico absorption, distribution, metabolism, excretion and toxicity parameters of synthesized compounds 3a-i were found within their acceptable limits. In acute toxicity study, none of synthesized 1,4-benzothiazepines showed any toxicity risks such as tremor, convulsion, lacrimation, sedation, increased or decreased motor activity, etc., in Wistar rats. The antihyperglycemic activity showed that synthesized 1,4-benzothiazepines (3a-i) reduced the elevated blood glucose level significantly in Wistar rats. $3 \mathrm{f}, 3 \mathrm{~h}$ and $3 \mathrm{i}$ with electronegative substitution at 7 position of benzothiazepine nucleus and at 2 ' position of phenyl ring, showed better antihyperglycemic activity as compared to other 1,4-benzothiazepines having weak electronegative substituent at the same position. The antihyperglycemic activity of synthesized 1,4-benzothiazepines was comparable with antihyperglycemic activity of metformin and CGP37157. The present study may be helpful in development of novel antidiabetic agents.
\end{abstract}

Key words: Diabetes, 1,4-benzothiazepine, Friedel-Craft acylation, S-alkylation, in silico absorption, distribution, metabolism, excretion and toxicity, antihyperglycemic activity

Diabetes mellitus is a clinical condition which accounts for about $90 \%$ of diagnosed diabetic cases and is characterized by increased blood glucose level due to impaired insulin secretion, insulin action, insulin resistance and excessive hepatic gluconeogenesis ${ }^{[1,2]}$. Without enough insulin, body cells cannot take up sufficient glucose, which results in elevation of blood glucose level. Such conditions over long period of time can harm major organs such as liver, kidney, nerves, eyes, heart and blood vessels. Impairment in activity of some of these organs may lead to serious complications and even death ${ }^{[3]}$.

Several classes of drugs are available to treat type- 2 diabetes such as insulin secretagogues (sulfonylureas and meglitinides), insulin sensitizers (thiazolidinediones and biguanides), glucose uptake blockers (acarbose and pramlintide), insulin and modified insulin's etc. Efficacy of these drugs is limited due to mechanism related side effects (i.e. hypoglycemia, weight gain, gastrointestinal

*Address for correspondence E-mail: drmeenatiwari@gmail.com

September-October 2021 distress etc.), inadequacy for use as monotherapy and development of tolerance ${ }^{[4-6]}$. Sulfonylureas lose efficacy as their prolonged use causes $\beta$-cell fatigue ${ }^{[4]}$. Thiazolidinediones banned in some countries due to serious cardiac ailments and bladder cancer ${ }^{[7,8]}$. Insulin secretagogues, when used as monotherapy stimulate insulin secretion under fasting condition and increase the risk of hypoglycemia ${ }^{[9]}$. Dipeptidyl peptidase-4 inhibitors or gliptins are also associated with pancreatitis as a risk ${ }^{[10-12]}$. Unfortunately, none of the oral hypoglycemic agent has been completely successful in controlling hyperglycemia and longterm complications. This provides an opportunity to

This is an open access article distributed under the terms of the Creative Commons Attribution-NonCommercial-ShareAlike 3.0 License, which allows others to remix, tweak, and build upon the work non-commercially, as long as the author is credited and the new creations are licensed under the identical terms

Accepted 17 October 2021

Revised 18 July 2021

Received 30 March 2020

Indian J Pharm Sci 2021;83(5):1033-1043 
medicinal chemists to explore other class of chemical compounds as novel antihyperglycemic agents which can treat type 2 diabetes effectively and safely.

Compounds from 1,4-benzothiazepine-2-one and 1, 5-benzothiazepine-2-one chemical classes have been reported to inhibit mitochondrial sodium calcium exchange (mNCE) activity ${ }^{[13]}$ (fig. 1). The mNCE inhibitors augment mitochondrial oxidative metabolism and stimulate glucose, stimulated insulin secretion in a glucose-dependent manner in rat islets and in a rat Insulinoma Cell Line-1 (INS-1) cell culture model ${ }^{[13-18]}$, thus, can be explored as antihyperglycemic agent.

Previously, a Comparative Molecular Field Analysis (CoMFA) of Benzothiazepines (BTZs) as antidiabetic agents has been reported by our group ${ }^{[19]}$. As an ongoing effort, the present study reported the synthesis of some 1,4-BTZs and their antihyperglycemic activity was evaluated in Wistar rats. CGP37157, a potent $\mathrm{mNCE}$ inhibitor of 1,4-BTZ class was also synthesized and evaluated for its antihyperglycemic activity. Metformin was taken and reference antidiabetic drug and the antihyperglycemic activity of synthesized 1,4-BTZs was compared against it.

In the drug development process, most of the developed drug molecules failed in the clinical phases due to their poor pharmacokinetics and toxicity ${ }^{[20]}$. Thus, it is always beneficial to point out such failures at early stage of drug development. In this study, a preliminary drug-likeness, in silico Absorption, Distribution, Metabolism, Excretion and Toxicity (ADME/T) profiling and toxicity risk assessments (i.e. Mutagenicity (MUT), Tumorigenicity (TUM), Irritant (IRR) and Reproductive Effects (RPRD)) studies were performed to determine the pharmacokinetic and toxicity profile of synthesized 1,4-BTZs.

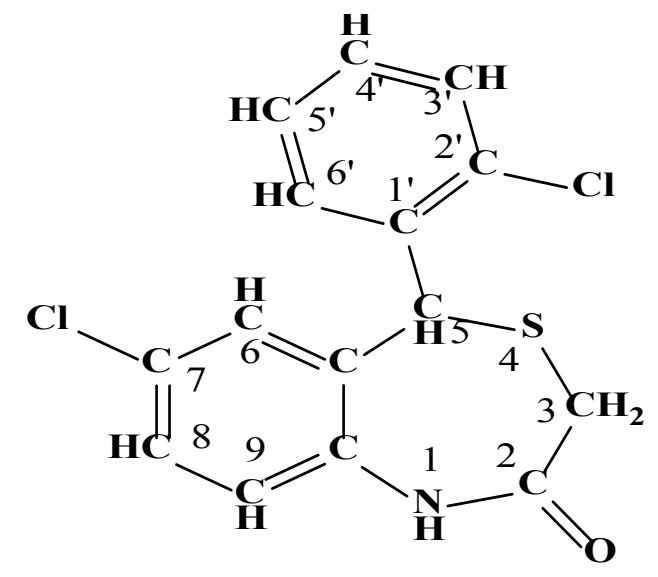

Fig. 1: Structure of CGP37157 
30 min. Crystals of substituted benzhydrols (2a-i) were collected by filtration and rinsed with small amount of cold water.

\section{Synthesis of 1,4-BTZs (3a-i):}

Basicalumina $(7.0 \mathrm{~g})$ was added toa mixtureof substituted benzhydrols $(3.75 \mathrm{mmol})$, methylthioglycolate $(15.0 \mathrm{mmol})$ and trifluoroacetic acid $(52.0 \mathrm{mmol})$ in a $50 \mathrm{ml}$ flat bottom flask and subjected to microwave irradiation for $7 \mathrm{~min}$ at full power $(800 \mathrm{~W})$. The mixture was extracted with chloroform. The extract was concentrated under vacuum, redissolved in $\mathrm{CH}_{2} \mathrm{Cl}_{2}$, washed with saturated aqueous sodium carbonate and brine and passed through anhydrous sodium sulphate. Finally, the extract was concentrated under vacuum to obtain the final product, which was further purified by column chromatography. The physicochemical properties and spectral data of synthesized 1,4-BTZs are as follows:

7-methoxy-5-phenylbenzo [1, 4] thiazepin-2 (1H, 3H, 5H)-one (3a): A light brown solid product was formed by microwave assisted S-alkylation of 2-amino-5methoxy benzhydrol; yield $(\%)=65.40$; mp: $164-169^{\circ}$; Retention Factor $\left(\mathrm{R}_{\mathrm{f}}\right)$ [hexane/acetone $\left.(8: 2)\right]=0.67 ; \lambda_{\max }$ (nm): 336.6, 273.4; $\log \mathrm{P}: 3.05$; IR (Potassium Bromide $\left.(\mathrm{KBr}), \mathrm{cm}^{-1}\right)$ : $3286(\mathrm{~N}-\mathrm{H}), 3040(\mathrm{C}-\mathrm{H}$, argon $(\mathrm{Ar}))$, $1681(\mathrm{C}=\mathrm{O}), 1533(\mathrm{C}=\mathrm{C}, \mathrm{Ar}), 1183(\mathrm{C}-\mathrm{O}-\mathrm{C}) ;{ }^{1} \mathrm{H}-\mathrm{NMR}$ (400 MHz, $\left.\mathrm{CDCl}_{3}, \mathrm{ppm}\right): \delta$ 7.33-7.09 (m, 6H, CH), 6.83 $(\mathrm{d}, \mathrm{J}=1.3 \mathrm{~Hz}, 1 \mathrm{H}, \mathrm{CH}), 6.78(\mathrm{dd}, \mathrm{J}=7.4,1.4 \mathrm{~Hz}, 1 \mathrm{H}, \mathrm{CH})$, 5.32 (s, 1H, NH), 5.08 (s, 1H, CH), 3.73 (s, 3H, Methyl Group $\left.\left(\mathrm{CH}_{3}\right)\right), 3.42(\mathrm{~d}, \mathrm{~J}=12.4 \mathrm{~Hz}, 1 \mathrm{H}, \mathrm{CH}), 3.23$ (d, $\mathrm{J}=12.5 \mathrm{~Hz}, 1 \mathrm{H}, \mathrm{CH})$; calculated molecular weight: 285.36; found (Electron Ionization Mass Spectrum (EIMS)) mass to charge ratio (m/z): $285.08(\mathrm{M}+$ peak), 286.09 (M+1 peak); molecular formula: $\mathrm{C}_{16} \mathrm{H}_{15} \mathrm{NO}_{2} \mathrm{~S}$, elemental analysis (\%), calculated: C, 67.34; H, 5.30; N, 4.91; S, 11.24; found: C, 67.18; H, 5.23; N, 4.98; S, 11.02 .

\section{5-(2-chlorophenyl)-7-methoxybenzo[1,4]thiazepin-} 2(1H, 3H, 5H)-one (3b): A white solid product was formed by microwave assisted S-alkylation of 2-amino5-methoxy-2'-chlorobenzhydrol; yield $(\%)=75.92, \mathrm{mp}$ : $179-185^{\circ}, \mathrm{R}_{\mathrm{f}}$ [hexane/acetone $\left.(8: 2)\right]=0.73 ; \lambda_{\max }(\mathrm{nm})$ : 339.2, 285.2; $\log$ P: 3.61; IR $\left(\mathrm{KBr}, \mathrm{cm}^{-1}\right): 3288(\mathrm{~N}-\mathrm{H})$, 3025 (C-H, Ar), $1657(\mathrm{C}=\mathrm{O}), 1498(\mathrm{C}=\mathrm{C}, \mathrm{Ar}), 1164$ (C-O-C), 1085 (C-Cl); ${ }^{1} \mathrm{H}-\mathrm{NMR}\left(400 \mathrm{MHz}, \mathrm{CDCl}_{3}\right.$, ppm): $\delta$ 7.78-7.02 (m, 7H, $\mathrm{CH}$ and $\mathrm{NH}), 6.19(\mathrm{dd}$, $\mathrm{J}=7.5,1.4 \mathrm{~Hz}, 1 \mathrm{H}, \mathrm{CH}), 5.11(\mathrm{~s}, 1 \mathrm{H}, \mathrm{CH}), 3.78(\mathrm{~s}, 3 \mathrm{H}$, $\left.\mathrm{CH}_{3}\right), 3.31$ (d, J=12.5 Hz, 1H, CH), 3.25 (d, J=12.5 Hz, $1 \mathrm{H}, \mathrm{CH}$ ); calculated molecular weight: 319.81 ; found
(EIMS) m/z: 319.04 (M+ peak), 321.04 (M+2 peak); molecular formula: $\mathrm{C}_{16} \mathrm{H}_{14} \mathrm{ClNO}_{2} \mathrm{~S}$, elemental analysis (\%), calculated: C, 60.09; H, 4.41; N, 4.38; S, 10.03; found: C, 59.98; H, 4.30; N, 4.32; S, 9.95 .

7-methyl-5-phenylbenzo $[1,4]$ thiazepin-2 $(1 \mathrm{H}, 3 \mathrm{H}$, 5H)-one (3c): A brown solid product was formed by microwave assisted S-alkylation of 2-amino-5methyl-benzhydrol; yield $(\%)=68.91 ; \mathrm{mp}: 170-175^{\circ}$; $\mathrm{R}_{\mathrm{f}}$ [hexane/acetone $\left.(8: 2)\right]=0.76 ; \lambda_{\max }(\mathrm{nm}): 235.4$, 312.4; $\log \mathrm{P}: 3.67$; IR (KBr, cm$\left.{ }^{-1}\right): 3292(\mathrm{~N}-\mathrm{H}), 3032$ (C-H, Ar), $1676(\mathrm{C}=\mathrm{O}), 1493(\mathrm{C}=\mathrm{C}, \mathrm{Ar}) ;{ }^{1} \mathrm{H}-\mathrm{NMR}(400$ $\left.\mathrm{MHz}, \mathrm{CDCl}_{3}, \mathrm{ppm}\right): \delta$ 7.32-7.23 (m, 1H, CH), 7.21$7.11(\mathrm{~m}, 7 \mathrm{H}, \mathrm{CH}), 6.94(\mathrm{~s}, 1 \mathrm{H}, \mathrm{NH}), 5.12(\mathrm{~s}, 1 \mathrm{H}, \mathrm{CH})$, $3.31(\mathrm{~d}, \mathrm{~J}=12.3 \mathrm{~Hz}, 1 \mathrm{H}, \mathrm{CH}), 3.29$ (d, J=12.5 Hz, 1H, $\mathrm{CH}), 2.43$ (s, 3H, $\mathrm{CH}_{3}$ ); calculated molecular weight: 269.36; found (EIMS) m/z: 269.09 (M+ peak), 270.09 (M+1 peak); molecular formula: $\mathrm{C}_{16} \mathrm{H}_{15} \mathrm{NOS}$, elemental analysis (\%), calculated: $\mathrm{C}, 71.34 ; \mathrm{H}, 5.61 ; \mathrm{N}, 5.20 ; \mathrm{S}$, 11.90; found: C, 71.08; H, 5.69; N, 4.98; S, 11.69.

\section{5-(2-chlorophenyl)-7-methylbenzo $[1,4]$ thiazepin-} 2(1H, 3H, 5H)-one (3d): A yellowish white solid product was formed by microwave assisted S-alkylation of 2-amino-5-methyl-2'-chlorobenzhydrol; yield $(\%)=78.39 ; \quad \mathrm{mp}: \quad 184-192^{\circ} ; \quad \mathrm{R}_{\mathrm{f}} \quad$ [hexane/acetone $(8: 2)]=0.69, \lambda_{\max }(\mathrm{nm}): 234.3,301.2 ; \log \mathrm{P}: 4.22$; IR $\left(\mathrm{KBr}, \mathrm{cm}^{-1}\right): 3288(\mathrm{~N}-\mathrm{H}), 3038(\mathrm{C}-\mathrm{H}, \mathrm{Ar}), 1651(\mathrm{C}=\mathrm{O})$, $1503(\mathrm{C}=\mathrm{C}, \mathrm{Ar}), 1078$ (C-Cl); ${ }^{1} \mathrm{H}-\mathrm{NMR}(400 \mathrm{MHz}$, $\left.\mathrm{CDCl}_{3}, \mathrm{ppm}\right): \delta$ 7.89-7.13 (m, 7H, CH), $5.43(\mathrm{~s}, 1 \mathrm{H}$, $\mathrm{NH}), 5.13(\mathrm{~s}, 1 \mathrm{H}, \mathrm{CH}), 3.42(\mathrm{~d}, \mathrm{~J}=12.5 \mathrm{~Hz}, 1 \mathrm{H}, \mathrm{CH}), 3.29$ (d, J=12.3 Hz, 1H, CH), $2.23\left(\mathrm{~s}, 3 \mathrm{H}, \mathrm{CH}_{3}\right)$; calculated molecular weight: 303.81; found (EIMS) m/z: 303.05 (M+ peak), 305.05 (M+2 peak); molecular formula: $\mathrm{C}_{16} \mathrm{H}_{14} \mathrm{ClNOS}$, elemental analysis (\%), calculated: $\mathrm{C}$, 63.25; H, 4.64; N, 4.61; S, 10.55; found: C, 63.12; H, $4.52 ; \mathrm{N}, 4.39 ; \mathrm{S}, 10.59$.

7-bromo-5-phenylbenzo [1,4] thiazepin-2 (1H, 3H, 5H)-one (3e): A white solid product was formed by microwave assisted S-alkylation of 2-amino-5bromo-benzhydrol; yield $(\%)=69.09$; mp: 176-183; $\mathrm{R}_{\mathrm{f}}[$ hexane/acetone $(8: 2)]=0.81 ; \lambda_{\max }(\mathrm{nm}): 265.2$, 295.3; $\log$ P: 3.57; IR $\left(\mathrm{KBr}, \mathrm{cm}^{-1}\right): 3289(\mathrm{~N}-\mathrm{H}), 3042$ (C-H, Ar), $1673(\mathrm{C}=\mathrm{O}), 1499(\mathrm{C}=\mathrm{C}, \mathrm{Ar}), 1045$ (C-Br); ${ }^{1} \mathrm{H}-\mathrm{NMR}\left(400 \mathrm{MHz}, \mathrm{CDCl}_{3}, \mathrm{ppm}\right): \delta 7.62-7.52(\mathrm{~m}, 2 \mathrm{H}$, $\mathrm{CH}$ and $\mathrm{NH}), 7.43$ (dd, J=7.5, $1.4 \mathrm{~Hz}, 1 \mathrm{H}, \mathrm{CH}), 7.29$ $7.13(\mathrm{~m}, 6 \mathrm{H}, \mathrm{CH}), 5.12(\mathrm{~s}, 1 \mathrm{H}, \mathrm{CH}), 3.42$ (d, J=12.5 $\mathrm{Hz}, 1 \mathrm{H}, \mathrm{CH}), 3.33$ (d, J=12.5 Hz, 1H, CH); calculated molecular weight: 334.23 ; found (EIMS) m/z: 334.98 (M+ peak), 332.98 (M-2 peak); molecular formula: $\mathrm{C}_{15} \mathrm{H}_{12}$ BrNOS; elemental analysis (\%), calculated: $\mathrm{C}$, 
53.90; H, 3.62; N, 4.19; S, 9.59; found: C, 53.56; H, $3.79 ; \mathrm{N}, 4.01 ; \mathrm{S}, 9.42$.

7-bromo-5-(2-chlorophenyl) benzo $[1,4]$ thiazepin2-(1H, 3H, 5H)-one (3f): A brownish white solid product was formed by microwave assisted S-alkylation of 2-amino-5-bromo-2'-chlorobenzhydrol; yield $(\%)=72.79 ; \quad \mathrm{mp}: \quad 188-195^{\circ} ; \quad \mathrm{R}_{\mathrm{f}}$ [hexane/acetone $(8: 2)]=0.71 ; \lambda_{\max }(\mathrm{nm}): 312.3,287.1 ; \log \mathrm{P}: 4.01 ; \mathrm{IR}$ $\left(\mathrm{KBr}, \mathrm{cm}^{-1}\right): 3291(\mathrm{~N}-\mathrm{H}), 3048(\mathrm{C}-\mathrm{H}, \mathrm{Ar}), 1681(\mathrm{C}=\mathrm{O})$, $1512(\mathrm{C}=\mathrm{C}, \mathrm{Ar}), 1095$ (C-Cl), 1060 (C-Br); ${ }^{1} \mathrm{H}-\mathrm{NMR}$ (400 MHz, $\left.\mathrm{CDCl}_{3}, \mathrm{ppm}\right): \delta 8.22(\mathrm{~s}, 1 \mathrm{H}, \mathrm{NH}), 7.46$ (d, J=1.6 Hz, 1H, CH), 7.33 (dd, J=7.5, $1.5 \mathrm{~Hz}, 1 \mathrm{H}$, $\mathrm{CH}), 7.32-6.22(\mathrm{~m}, 5 \mathrm{H}, \mathrm{CH}), 5.13(\mathrm{~s}, 1 \mathrm{H}, \mathrm{CH}), 3.43$ (d, J=12.3 Hz, 1H, CH), 3.33 (d, J=12.3 Hz, 1H, CH); calculated molecular weight: 368.68; found (EIMS) m/z: 368.94 (M+ peak), 366.94 (M-2 peak); molecular formula: $\mathrm{C}_{15} \mathrm{H}_{11} \mathrm{BrClNOS}$; elemental analysis (\%), calculated: C, 48.87; H, 3.01; N, 3.80; S, 8.70, found: C, 48.45; H, 2.93; N, 3.59; S, 8.55.

7-fluoro-5-phenylbenzo [1,4] thiazepin-2(1H, 3H, 5H)-one (3g): A white solid product was formed by microwave assisted S-alkylation of 2-amino-5fluoro-benzhydrol; yield (\%)=72.79; mp: 169-176 $; \mathrm{R}_{\mathrm{f}}$ [hexane/acetone (8:2)] $=0.59 ; \lambda_{\max }(\mathrm{nm}): 323.0,239.4$; $\log$ : 2.89; IR (KBr, cm $\left.{ }^{-1}\right): 3261(\mathrm{~N}-\mathrm{H}), 3043$ (C-H, Ar), $1656(\mathrm{C}=\mathrm{O}), 1534(\mathrm{C}=\mathrm{C}, \mathrm{Ar}), 1195(\mathrm{C}-\mathrm{F})$; ${ }^{1} \mathrm{H}-\mathrm{NMR}$ (400 MHz, $\left.\mathrm{CDCl}_{3}, \mathrm{ppm}\right): \delta 7.78(\mathrm{~s}, 1 \mathrm{H}, \mathrm{NH}), 7.23-7.13$ (m, 7H, CH), 7.02 (d, J=7.8, $1.3 \mathrm{~Hz}, 1 \mathrm{H}, \mathrm{CH}), 5.13$ (s, 1H, CH), $3.44(\mathrm{~d}, \mathrm{~J}=12.4 \mathrm{~Hz}, 1 \mathrm{H}, \mathrm{CH}), 3.33$ (dt, $\mathrm{J}=12.4 \mathrm{~Hz}, 1 \mathrm{H}$ ); calculated molecular weight: 273.33; found (EIMS) m/z: $273.06(\mathrm{M}+$ peak), $274.03(\mathrm{M}+1$ peak); molecular formula: $\mathrm{C}_{15} \mathrm{H}_{12} \mathrm{FNOS}$; elemental analysis (\%), calculated: $\mathrm{C}, 65.91 ; \mathrm{H}, 4.43 ; \mathrm{N}, 5.12 ; \mathrm{S}$, 11.73; found: C, 65.82; H, 4.21; N, 4.99; S, 11.34.

5-(2-chlorophenyl)-7-fluorobenzo $[1,4]$ thiazepin2(1H, 3H, 5H)-one (3h): A yellow solid product was formed by microwave assisted S-alkylation of 2-amino5-fluoro-2'-chlorobenzhydrol; yield $(\%)=83.34 ; \mathrm{mp}$ : $182-190^{\circ} ; \mathrm{R}_{\mathrm{f}}$ [hexane/acetone $\left.(8: 2)\right]=0.68 ; \lambda_{\max }(\mathrm{nm})$ : 287.3, 253.4; $\log \mathrm{P}: 3.45$; IR $\left(\mathrm{KBr}, \mathrm{cm}^{-1}\right): 3235(\mathrm{~N}-\mathrm{H})$, 3053 (C-H, Ar), 1645 (C=O), $1543(\mathrm{C}=\mathrm{C}, \mathrm{Ar}), 1089$ (C-Cl), 1184 (C-F); ${ }^{1} \mathrm{H}-\mathrm{NMR}$ (400 MHz, $\left.\mathrm{CDCl}_{3}, \mathrm{ppm}\right)$ : $\delta 7.92(\mathrm{~s}, 1 \mathrm{H}, \mathrm{NH}), 7.21$ (dtdd, J=16.4, 14.5, 7.4, $1.6 \mathrm{~Hz}$, 5H, CH), 7.09-6.92 (m, 2H, CH), $5.11(\mathrm{~s}, 1 \mathrm{H}, \mathrm{CH}), 3.34$ $(\mathrm{d}, \mathrm{J}=12.4 \mathrm{~Hz}, 1 \mathrm{H}, \mathrm{SH})$; calculated molecular weight: 307.77; found (EIMS) m/z: 307.2 (M+ peak), 309.02 $\left(\mathrm{M}+2\right.$ peak); molecular formula: $\mathrm{C}_{15} \mathrm{H}_{11}$ ClFNOS; elemental analysis (\%): C, 58.54; H, 3.60; N, 4.55; S, 10.42; found: C, 58.44; H, 3.34; N, 4.34; S, 10.32 . 7-fluoro-5-(2-fluorophenyl)benzo $[1,4]$ thiazepin2(1H, 3H, 5H)-one (3i): A white solid product was formed by microwave assisted S-alkylation of 2-amino5-fluoro-2'-fluorobenzhydrol; yield $(\%)=82.43$; $\mathrm{mp}$ : $193-200^{\circ} ; \mathrm{R}_{\mathrm{f}}$ [hexane/acetone $\left.(8: 2)\right]=0.65 ; \lambda_{\max }(\mathrm{nm})$ : 245.3, 310.4; $\log \mathrm{P}: 3.05$; IR (KBr, $\left.\mathrm{cm}^{-1}\right): 3298(\mathrm{~N}-\mathrm{H})$, $3037(\mathrm{C}-\mathrm{H}, \mathrm{Ar}), 1679(\mathrm{C}=\mathrm{O}), 1530(\mathrm{C}=\mathrm{C}, \mathrm{Ar}), 1089$ (C-Cl), 1232(C-F); ${ }^{1} \mathrm{H}-\mathrm{NMR}$ (400 MHz, $\left.\mathrm{CDCl}_{3}, \mathrm{ppm}\right)$ : $\delta 7.58(\mathrm{~s}, 1 \mathrm{H}, \mathrm{NH}), 7.19(\mathrm{dd}, \mathrm{J}=9.9,7.5,6.2,3.1 \mathrm{~Hz}$, 4H, C-H, Ar), 7.11-6.97 (m, 3H, C-H, Ar), 5.11 (s, 1H, $\mathrm{CH}), 3.43(\mathrm{~d}, \mathrm{~J}=12.4 \mathrm{~Hz}, 1 \mathrm{H}, \mathrm{CH}), 3.32$ (d, J=12.4 Hz, $1 \mathrm{H}, \mathrm{CH})$; calculated molecular weight: 291.32; found (EIMS) m/z: 291.05 (M+ peak), 292.06 (M+1 peak); molecular formula: $\mathrm{C}_{15} \mathrm{H}_{11} \mathrm{~F}_{2} \mathrm{NOS}$; elemental analysis (\%), calculated: C, 61.84; H, 3.81; N, 4.81; S, 11.01; found: C, 61.34; H, 3.43; N, 4.45; S, 10.94 .

7-chloro-5-(2-chlorophenyl)-1,5-dihydro-4,1benzothiazepin-2(3H)-one (CGP37157): A yellowish white solid product was formed by microwave assisted S-alkylation of 2-amino-5, 2'dichloro benzhydrol; yield $(\%)=88.34 ; \mathrm{mp}: 125-130^{\circ} ; \mathrm{R}_{\mathrm{f}}$ [hexane/acetone $(8: 2)]=0.80, \lambda_{\text {max }}(\mathrm{nm}): 235.5,287.3 ; \log \mathrm{P}: 3.85$; IR $\left(\mathrm{KBr}, \mathrm{cm}^{-1}\right): 3298(\mathrm{~N}-\mathrm{H}), 3037(\mathrm{C}-\mathrm{H}, \mathrm{Ar}), 1679(\mathrm{C}=\mathrm{O})$, $1530(\mathrm{C}=\mathrm{C}, \mathrm{Ar}), 1089$ (C-Cl); ${ }^{1} \mathrm{H}-\mathrm{NMR}(400 \mathrm{MHz}$, $\left.\mathrm{CDCl}_{3}, \mathrm{ppm}\right): \delta 7.90(\mathrm{~s}, 1 \mathrm{H}, \mathrm{NH}), 7.43(\mathrm{~d}, \mathrm{~J}=1.4 \mathrm{~Hz}$, $1 \mathrm{H}, \mathrm{CH}), 7.33-6.89$ (m, 6H, CH), $5.12(\mathrm{~s}, 1 \mathrm{H}, \mathrm{CH})$, $3.42(\mathrm{~d}, \mathrm{~J}=12.3 \mathrm{~Hz}, 1 \mathrm{H}, \mathrm{CH}), 3.32(\mathrm{~d}, \mathrm{~J}=12.5 \mathrm{~Hz}$, $1 \mathrm{H}, \mathrm{CH})$; calculated molecular weight: 324.22 ; found (EIMS) m/z: 324.22(M+ peak), 322.99 (M-2 peak); molecular formula: $\mathrm{C}_{15} \mathrm{H}_{11} \mathrm{Cl}_{2} \mathrm{NOS}$; elemental analysis (\%), calculated: C, 55.57; H, 3.42; N, 4.32; S, 9.89, found: C, 55.65; H, 3.58; N, 4.61; S, 9.64.

\section{Drug-likeness and in silico ADME/T study:}

Drug-likeness of synthesized 1-4 BTZs was measured as per Lipinski's Rule of Five (ROF) and Jorgensen's Rule of Three (ROT), respectively ${ }^{[22,23]}$. As per Lipinski's ROF the molecule should have molecular weight $<500$, octanol/water partition coefficient $(\log \mathrm{P})<5$, number of Hydrogen Bond Donor (HBD) $\leq 5$, number of Hydrogen Bond Acceptor $(\mathrm{HBA}) \leq 10$, while as per Jorgensen's ROT the molecule should have aqueous solubility (Log S) $>-5.7$, predicted apparent Caco-2 cell permeability (Caco-2) $>22 \mathrm{~nm} / \mathrm{s}$, number of primary metabolites (\#metformin) $<7$. Molecules with a Polar Surface Area (PSA) less than $140 \AA^{2}$ (angstroms squared) have good cell membranes permeating property and also possesses better drug-likeness ${ }^{[24]}$. In silico prediction of ADME properties and toxicity risks i.e. MUT and TUM, IRR and RPRD of compounds was done by Qikprop module 
of Schrodinger suite $2010^{[25-28]}$ and DataWarrior ${ }^{[29-31]}$, respectively.

\section{Biological evaluation:}

Male Wistar rats weighing between 150-200 g were used for screening of antihyperglycemic activity of synthesized 1,4-BTZs ${ }^{[32,33]}$. All animals (Wistar rats) were maintained under $12 \mathrm{~h}$ light and $12 \mathrm{~h}$ dark cycle at $25^{\circ}$ and given standard pellet diet (supplied by Godrej Agrowet Ltd., Sanwer road, Indore) and water ad libitum. The acclimatized animals were kept fasting for $24 \mathrm{~h}$ before experiment. All animal experiments were done using the protocols as per Institutional Animal Ethics Committee.

\section{Acute toxicity study:}

To determine the acute toxicity of synthesized 1,4-BTZs, different doses of 3a-i ranges from $100-500 \mathrm{mg} / \mathrm{kg}$ of body weight were administered to ten groups (contain three rats each) of Wistar rats ${ }^{[34]}$. Control group (also contains three rats) received $0.25 \%$ carboxymethyl cellulose suspension only. All animal groups were observed for $24 \mathrm{~h}$ to detect any signs of acute toxicity such as tremor, convulsion, lacrimation, sedation, increased or decreased motor activity etc. No such signs were observed even after $24 \mathrm{~h}$. Hence, the final dose of test compounds was fixed as $500 \mathrm{mg} / \mathrm{kg} .1 / 10^{\text {th }}$ of the final dose i.e. $50 \mathrm{mg} / \mathrm{kg}$ of body weight was taken as screening dose for evaluation of antihyperglycemic activity ${ }^{[32,35,36]}$.

\section{Antihyperglycemic activity:}

The experimental animals were divided into four groups. Group I (control), II (diabetic control) and III contained six rats each. Group IV was subdivided in 10 subgroups (S1-S10), which also consisted of six rats each. Alloxan monohydrate (dissolved in sterile normal saline) was injected Intraperitoneally (i.p.) to all animal groups (except group I) at a dose of $120 \mathrm{mg} / \mathrm{kg}$ body weight. All animal groups kept for next $24 \mathrm{~h}$ on $10 \%$ glucose solution to overcome early hypoglycemic phase. Serum glucose level was checked after $72 \mathrm{~h}$. Animals with serum glucose levels $>250 \mathrm{mg} / \mathrm{dl}$ were considered diabetic and were used for further study ${ }^{[36,37]}$. Group I and II animals were given vehicle only. Animals of group III were orally fed $0.25 \%$ carboxymethyl cellulose suspension ofmetformin (50 $\mathrm{mg} / \mathrm{kg}$ bodyweight) ${ }^{[38]}$. Subgroups (S1-S10) of group IV received $3 \mathrm{a}-\mathrm{i}$ and CGP37157 $(50 \mathrm{mg} / \mathrm{kg}$ bodyweight), respectively, as $0.25 \%$ carboxymethyl cellulose oral suspension.
Fasting blood samples were collected from tail vein after $72 \mathrm{~h}$ of alloxan treatment (prior to administration of synthesized 1,4-BTZs) ${ }^{[39]}$. For biochemical study, animals were administered the same dose of synthesized 1,4-BTZs once daily for $15 \mathrm{~d}$ (counted after $72 \mathrm{~h}$ of alloxan treatment). Blood glucose level was measured on $1^{\text {st }} \mathrm{d}, 5^{\text {th }} \mathrm{d}, 10^{\text {th }} \mathrm{d}$ and $15^{\text {th }} \mathrm{d}$ by blood glucose monitoring system (Roche Diagnostics Corporation). Data obtained from antihyperglycemic activity was expressed as mean \pm Standard Error of Mean (SEM) and analyzed by Analysis of Variance (ANOVA) and post hoc Dunnett's t-test. Differences between groups were considered significant at $\mathrm{p}<0.05$ levels.

\section{RESULTS AND DISCUSSION}

In the previous study, a CoMFA was carried out on a series of BTZs as antidiabetic agents ${ }^{[13]}$, which resulted in the generation of various electrostatic and steric fields around the BTZ template (fig. 2). Subsequently, several BTZs were designed as potent antidiabetic agents ${ }^{[19]}$. In the present study, 1,4-BTZs, (3a-i) were synthesized and evaluated for antihyperglycemic activity.

Compounds 3a-i was synthesized via three step processes (fig. 3). The first step involved Friedel-Crafts acylation of para-substituted anilines, which resulted in the formation of substituted benzophenones. IR bands at $3411(\mathrm{~N}-\mathrm{H}), 3314(\mathrm{C}-\mathrm{H}, \mathrm{Ar}), 1613(\mathrm{C}=\mathrm{O}), 1532$ $(\mathrm{C}=\mathrm{C}, \mathrm{Ar}) \mathrm{cm}^{-1}$ confirmed the synthesis of substituted benzophenones.

The second step was reduction of substituted benzophenones to corresponding alcohols by $\mathrm{NaBH}_{4}$. $\mathrm{NaBH}_{4}$ (a complex metal hydride) is useful reagent for reduction of aldehydes and ketones ${ }^{[40]}$. The formation of benzhydrols was confirmed by IR through disappearance of ketone band at $1680 \mathrm{~cm}^{-1}$ and appearance of $-\mathrm{OH}$ band at $3400-3600 \mathrm{~cm}^{-1}$.

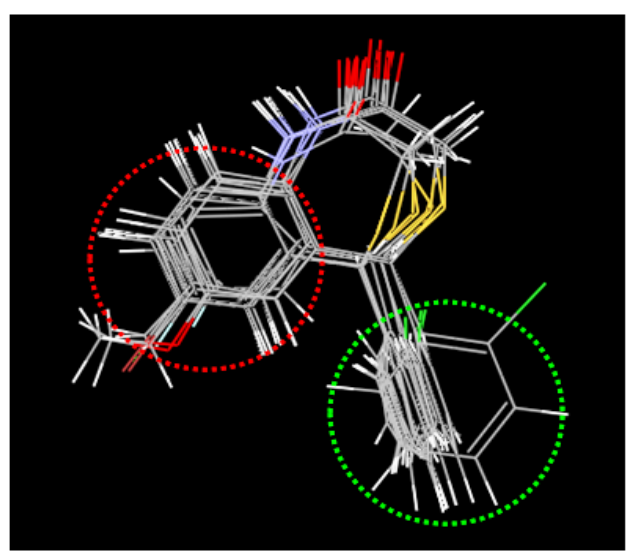

Fig. 2: BTZ template with steric and electrostatic fields developed by CoMFA 


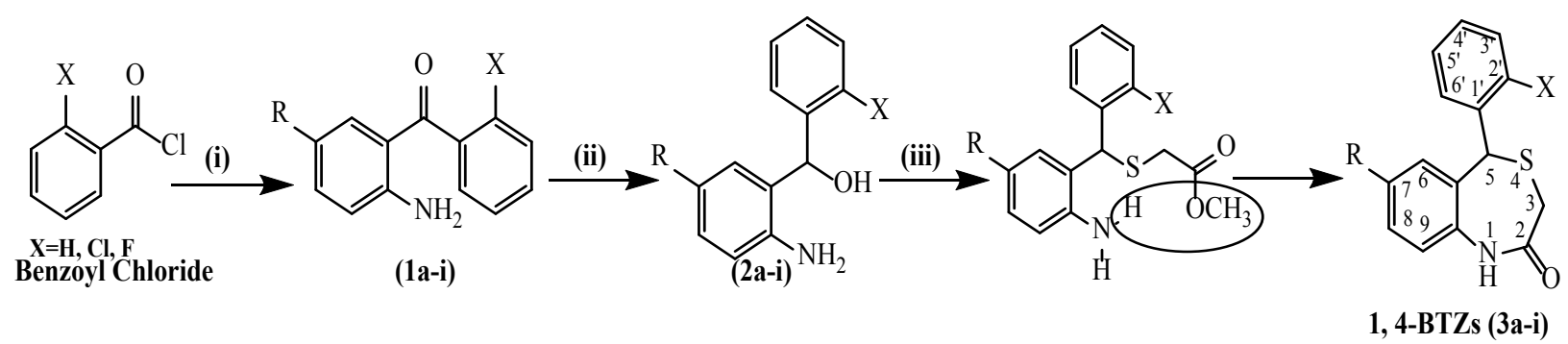

Fig. 3: Reaction scheme for synthesis of 3a-i. Reagent and conditions: (i) p-substituted anilines $\left(\mathrm{R}=\mathrm{OCH}_{3}, \mathrm{CH}_{3}, \mathrm{Br}, \mathrm{F}\right), \mathrm{ZnCl}, \mathrm{reflux}$ (ii) $\mathrm{NaBH}_{4}, 10 \% \mathrm{HCl}, \mathrm{CH}_{3} \mathrm{OH}$, stirring (iii) methylthioglycolate, trifluoroacetic acid, basic alumina, microwave irradiation. 1a, 2a, 3a: $\mathrm{R}=\mathrm{OCH}_{3}, \mathrm{X}=\mathrm{H}$; 1b, 2b, 3b: $\mathrm{R}=\mathrm{OCH}_{3}, \mathrm{X}=\mathrm{Cl}$; 1c, 2c, 3c: $\mathrm{R}=\mathrm{CH}_{3}, \mathrm{X}=\mathrm{H}$; 1d, 2d, 3d: $\mathrm{R}=\mathrm{CH}, \mathrm{X}=\mathrm{Cl}$; 1e, 2e, 3e: $\mathrm{R}=\mathrm{Br}, \mathrm{X}=\mathrm{H}$; 1f, 2f, 3f: $R=B r, X=C l ; 1 g, 2 g, 3 g: R=F, X=H ; 1 h, 2 h, 3 h: R=F, X=C l ; 11,2 i, 3 i: R=F, X=F$

The final step was microwave-assisted S-alkylation of substituted benzhydrols. The conventional reaction for synthesis of benzothiazepinones takes approximately $72 \mathrm{~h}$ to complete via S-alkylation of thioglycolic acid with 2-aminobenzhydrol in presence of $\mathrm{HCl}$, followed by cyclization. In contrast, microwave irradiation method takes less time in completion of reaction (about 20-25 min) with higher yield. Synthesized 1,4-BTZs were purified by column chromatography on a silica gel column using a mixture of hexane and acetone as an eluent. CGP37157 was also synthesized according to the same reaction scheme and purified by column chromatography.

Formation of the 1,4-BTZs was confirmed by above spectral data. IR bands between 3010-3050 $\mathrm{cm}^{-1}$ (C-H, Ar) and 1600-1475 $\mathrm{cm}^{-1}(\mathrm{C}=\mathrm{C}, \mathrm{Ar})$ confirmed the presence of aromatic rings. Bands near $3300 \mathrm{~cm}^{-1}$ $(\mathrm{N}-\mathrm{H})$ and between $1680-1630 \mathrm{~cm}^{-1}(\mathrm{C}=\mathrm{O})$ confirmed the presence of lactam ring with amide functionality. Aryl chlorides, aryl fluorides and aryl bromides were confirmed by bands between 1100-1035 $\mathrm{cm}^{-1}$. $1250-1100 \mathrm{~cm}^{-1}$ and $1075-1030 \mathrm{~cm}^{-1}$, respectively ${ }^{[41]}$. Chemical shift values $(\delta, \mathrm{ppm})$ 3.36-3.46 (doublet) for $\mathrm{CH}_{2}$ group at position 3, 5.10 (singlet) for $\mathrm{CH}$ group at position 5, 5-8.5 (singlet) for $1-\mathrm{NH}$ and 6.5 -7.5 (multiplet) for CH group at position 6, 8, 9, 2', 3', 4' and 5' confirmed that 1,4-BTZs have been synthesized. $3 \mathrm{a}$ and $3 \mathrm{~b}$ showed chemical shift value for 7-methoxy substituent between $\delta$ 3.79-3.85 whereas $3 \mathrm{c}$ and $3 \mathrm{~d}$ showed specific chemical shift values for 7-methyl group and 1-NH group between $\delta$ 2.30-2.44 and $\delta$ $5.47-7.00$, respectively. The molecular weight of the synthesized 1,4-BTZs was confirmed by mass spectrum. The presence of aryl halides was confirmed by presence of $M+2$ peak in the mass spectrum of $3 b, 3 d, 3 f$ and $3 \mathrm{~h}$. Further, agreement between calculated and found values of elemental analysis confirmed the formation of 3a-i and CGP37157. Synthesized 1,4-BTZs were searched for structural similarity on various chemical databases like ChemSpider, Chemfrog, iScienceSearch and ChemDB portal. No similar structure was found for any of the synthesized compound (Table 1).

Compounds with fewer (and preferably no) violations of Lipinski's ROF and Jorgensen's ROT are more likely to be orally available and the synthesized compounds 3a-i have shown no violations of these rules (Table 2). All the synthesized 1,4-BTZs showed values of ADME parameters i.e. $\log \mathrm{S}$, \#metformin (number of metabolic reactions), Central Nervous System (CNS) activity, Caco-2 permeability (permeability through intestinal epithelium), ability to cross Blood Brain Barrier ( $\operatorname{LogBB})$, permeability through skin ( $\operatorname{LogKp})$, serum protein binding (LogKhsa) and Percent Human Oral Absorption (\% HOA), within reference limits (Table 3). In silico toxicity study showed that all synthesized compounds have low toxicity risks for MUT, TUM, IRR and RPRD (Table 4).

Administration of alloxan $(120 \mathrm{mg} / \mathrm{kg}$, i.p.) led to 1.5 -fold elevation of fasting blood glucose level of Wistar rats, which was maintained over a period of 15 d. $2 \mathrm{w}$ administration of various derivatives led to dose dependent fall in elevated blood glucose level in Wistar rats. Effect reach maximum in $15 \mathrm{~d}$ of administration. Vehicle controlled animals had stable blood glucose level while diabetic animals which were treated with 3a-i showed significant reduction in elevated blood glucose level during $15 \mathrm{~d}$ (Table 5).

Percent reduction in elevated blood glucose level $(\% \mathrm{R})$ was calculated for individual compounds using the following formula:

$\% \mathrm{R}=$ Initial blood glucose level (on d 1)-Final blood glucose level (on d 15)/Initial blood glucose level (on $\mathrm{d} 1) \times 100$

On the basis of $\% \mathrm{R}$, a comparison was made between all experimental groups. CGP37157, which contains 
$3 a$<smiles>COc1ccc2c(c1)C(c1ccccc1)SCC(=O)N2</smiles>

$3 \mathrm{~b}$

$3 c$

$3 d$<smiles>Cc1ccc2c(c1)C(c1ccccc1)SCC(=O)N2</smiles><smiles>Cc1ccc2c(c1)C(c1ccccc1Cl)SCC(=O)N2</smiles><smiles>O=C1CSC(c2ccccc2)c2cc(Br)ccc2N1</smiles>

$3 e$
ChemSpider: 0 hit Chemfrog: 0 hit iScienceSearch: 0 hit ChemDB portal: 0 hit

ChemSpider: 0 hit Chemfrog: 0 hit iScienceSearch: 0 hit ChemDB portal: 0 hit
ChemSpider: 0 hit Chemfrog: 0 hit iScienceSearch: 0 hit ChemDB portal: 0 hit
ChemSpider: 0 hit Chemfrog: 0 hit iScienceSearch: 0 hit ChemDB portal: 0 hit 
<smiles>O=C1CSC(c2ccccc2Cl)c2cc(Br)ccc2N1</smiles><smiles>O=C1CSC(c2ccccc2)c2cc(F)ccc2N1</smiles><smiles>O=C1CSC(c2ccccc2Cl)c2cc(F)ccc2N1</smiles>

ChemSpider: 0 hit Chemfrog: 0 hit iScienceSearch: 0 hit ChemDB portal: 0 hit

ChemSpider: 0 hit Chemfrog: 0 hit iScienceSearch: 0 hit ChemDB portal: 0 hit

$3 i$<smiles>O=C1CSC(c2ccccc2F)c2cc(F)ccc2N1</smiles>

ChemSpider: 0 hit Chemfrog: 0 hit iScienceSearch: 0 hit ChemDB portal: 0 hit

Synthesized compounds were searched on PubChem, ChemSpider, Chemfrog, iScienceSearch and ChemDB portal databases for similarity

$\mathrm{Cl}$ (chloro) group at 7 and 2' position, showed highest $\% \mathrm{R}$ value, which was comparable with \% $\mathrm{R}$ value of metformin. $3 \mathrm{~h}$ and $3 \mathrm{i}$ with $\mathrm{F}$ (flouro) group was equipotent as CGP37157. As the $\mathrm{F}$ was replaced with $\mathrm{Br}$ (bromo) (3e and 3f), \% R value was decreased due less electronegativity of $\mathrm{Br}$ as compared to $\mathrm{F}$. As the $\mathrm{Cl}$ at 7 position was replaced with methoxy $\left(\mathrm{OCH}_{3}\right)$ and $\mathrm{CH}_{3}, \% \mathrm{R}$ was reduced (3a, 3b, 3c and 3d). Removal of $\mathrm{Cl}$ at 2' position resulted in less potent compounds (3a: $31.4 \%, 3 \mathrm{c}: 29.8 \%$ and $3 \mathrm{e}: 34.3 \%$ ) as compared to 
TABLE 2: DRUG LIKENESS STUDY OF SYNTHESIZED COMPOUNDS

\begin{tabular}{lccccccc}
\hline Compound & Molecular weight & HBD & HBA & LogP & PSA & ROF & ROT \\
\hline 3a & 285.36 & 1 & 3.75 & 3.234 & 46.179 & 0 & 0 \\
3b & 319.805 & 1 & 3.75 & 3.524 & 46.156 & 0 & 0 \\
3c & 269.361 & 1 & 3 & 3.189 & 38.908 & 0 & 0 \\
3d & 303.806 & 1 & 3 & 3.628 & 38.914 & 0 & 0 \\
3e & 334.23 & 1 & 3 & 3.53 & 38.896 & 0 & 0 \\
3f & 368.675 & 1 & 3 & 3.972 & 38.853 & 0 & 0 \\
3g & 273.324 & 1 & 3 & 3.113 & 38.893 & 0 & 0 \\
3h & 307.769 & 1 & 3 & 3.564 & 38.865 & 0 & 0 \\
3i & 291.315 & 1 & 3 & 3.278 & 38.844 & 0 & 0 \\
CGP37157 & 324.224 & 1 & 3 & 3.891 & 38.835 & 0 & 0 \\
Metformin & 129.164 & 5 & 3.5 & -0.746 & 91.858 & 0 & 0 \\
RR & $<500$ & $<5$ & $<10$ & -2 to 6.5 & $<140$ & $<4$ & $<3$ \\
\hline
\end{tabular}

Note: ROF: Lipinski's rule of five violations; ROT: Jorgensen's rule of three violations; RR: Reference range for $95 \%$ of known drugs

TABLE 3: In silico ADME PROFILING OF SYNTHESIZED 1, 4-BTZs

\begin{tabular}{llcllllll}
\hline Compound & Log S & CNS & Caco-2 & LogBB & LogKp & LogKhsa & \% HOA & \#Metformin \\
\hline 3a & -4.298 & 0 & 2157.59 & -0.03 & -1.789 & 0.29 & 100 & 5 \\
3b & -4.499 & 1 & 2212.27 & 0.102 & -1.848 & 0.346 & 100 & 5 \\
3c & -4.261 & 1 & 2156.17 & 0.055 & -1.88 & 0.373 & 100 & 5 \\
3d & -4.656 & 1 & 2199.37 & 0.169 & -1.963 & 0.454 & 100 & 5 \\
3e & -4.507 & 1 & 2156.73 & 0.248 & -1.857 & 0.349 & 100 & 4 \\
3f & -5.212 & 1 & 2167.57 & 0.317 & -1.94 & 0.467 & 100 & 4 \\
3g & -3.982 & 1 & 2157.12 & 0.19 & -1.826 & 0.249 & 100 & 4 \\
3h & -4.49 & 1 & 2211.75 & 0.296 & -1.886 & 0.343 & 100 & 4 \\
3i & -4.197 & 1 & 2165.02 & 0.243 & -1.881 & 0.272 & 100 & 4 \\
CGP37157 & -4.968 & 1 & 2146.06 & 0.358 & -1.97 & 0.425 & 100 & 4 \\
Metformin & -0.556 & -2 & 211.107 & -1.137 & -6.414 & -0.934 & 64.179 & 2 \\
RR & -6.5 to 0.5 & -2 to 2 & $<25$ poor & -3 to 1.2 & -8 to -1 & \pm 1.5 & $<25$ poor & 1 to 8 \\
\hline
\end{tabular}

RR: Reference range for $95 \%$ of known drugs

TABLE 4: In silico TOXICITY RISK ASSESSMENT OF SYNTHESIZED 1,4-BTZs

\begin{tabular}{llllc}
\hline \multirow{2}{*}{ Compound } & & \multicolumn{2}{c}{ Toxicity risks } & \\
\cline { 2 - 5 } & MUT & TUM & RPRD & None \\
Ia & None & None & None & None \\
$3 \mathrm{~b}$ & None & None & None & None \\
$3 \mathrm{c}$ & None & None & None & None \\
$3 \mathrm{~d}$ & None & None & None & None \\
$3 f$ & None & None & None & None \\
$3 \mathrm{~g}$ & None & None & None & None \\
$3 \mathrm{~h}$ & None & None & None & None \\
$3 \mathrm{i}$ & None & None & None & None \\
CGP37157 & None & None & None & High \\
Metformin & None & None & None & None
\end{tabular}

MUT: mutagenicity; TUM: tumorigenicity; RPRD: reproductive effects; IRR: irritant

compounds containing 2' $\mathrm{Cl}$ substitution (3b: $33.0 \%$, $3 \mathrm{~d}: 32.7 \%$ and $3 \mathrm{f}: 42.4 \%$ ). Antidiabetic activity of the synthesized 1,4-BTZs at different intervals is presented by a bar graph (fig. 4). The study suggested that compounds containing electron withdrawing groups at position 7 and 2' were more active than compounds having electron donating groups at same position. 
TABLE 5: EFFECT OF 1,4-BTZS ON BLOOD GLUCOSE LEVEL OF WISTAR RATS AT DIFFERENT INTERVALS

\begin{tabular}{|c|c|c|c|c|c|c|c|c|}
\hline \multicolumn{9}{|c|}{ Average Blood glucose level (mg/dl)* } \\
\hline Group & Subgroup & Animal treatment & Before alloxan treatment & d 1 & d 5 & d 10 & d 15 & $\% R$ \\
\hline I & - & Control & $151.3 \pm 1.1$ & $154.9 \pm 0.4$ & $155.5 \pm 1.3$ & $143.4 \pm 1.2$ & $152.9 \pm 1.1$ & 1.3 \\
\hline II & - & Diabetic control & $155.7 \pm 0.9$ & $253.1 \pm 0.5$ & $248.8 \pm 1.6$ & $240.0 \pm 1.0$ & $220.6 \pm 0.9$ & 12.9 \\
\hline III & - & Metformin & $145.4 \pm 0.5$ & $285.2 \pm 1.4$ & $222.2 \pm 0.5$ & $178.9 \pm 1.9$ & $144.3 \pm 2.3$ & 49.4 \\
\hline \multirow{10}{*}{ IV } & S1 & $3 a$ & $154.3 \pm 1.2$ & $253.3 \pm 1.8$ & $223.9 \pm 0.8$ & $198.7 \pm 2.3$ & $173.7 \pm 0.5$ & 31.4 \\
\hline & S2 & $3 b$ & $154.2 \pm 1.1$ & $260.5 \pm 2.2$ & $216.4 \pm 0.6$ & $198.3 \pm 2.5$ & $174.4 \pm 1.3$ & 33.0 \\
\hline & S3 & $3 c$ & $158.7 \pm 1.2$ & $257.1 \pm 2.0$ & $213.7 \pm 0.8$ & $198.7 \pm 1.4$ & $180.3 \pm 1.9$ & 29.8 \\
\hline & S4 & $3 d$ & $159.4 \pm 0.8$ & $255.1 \pm 1.1$ & $202.8 \pm 0.6$ & $189.3 \pm 0.9$ & $171.7 \pm 1.5$ & 32.7 \\
\hline & S5 & $3 e$ & $154.8 \pm 0.5$ & $259.7 \pm 1.2$ & $208.5 \pm 1.1$ & $184.7 \pm 0.8$ & $170.5 \pm 0.8$ & 34.3 \\
\hline & S6 & $3 f$ & $148.3 \pm 1.5$ & $268.3 \pm 1.8$ & $210.0 \pm 1.0$ & $187.2 \pm 2.0$ & $154.5 \pm 0.7$ & 42.4 \\
\hline & S7 & $3 g$ & $150.2 \pm 1.3$ & $278.3 \pm 1.9$ & $205.3 \pm 1.2$ & $189.4 \pm 2.1$ & $170.9 \pm 1.7$ & 38.6 \\
\hline & S8 & $3 \mathrm{~h}$ & $152.1 \pm 0.9$ & $276.7 \pm 0.9$ & $219.5 \pm 0.9$ & $184.7 \pm 0.9$ & $153.7 \pm 0.9$ & 44.5 \\
\hline & S9 & $3 i$ & $149.2 \pm 0.4$ & $273.5 \pm 0.5$ & $212.4 \pm 0.5$ & $180.7 \pm 0.8$ & $148.2 \pm 1.5$ & 45.8 \\
\hline & S10 & CGP37157 & $140.3 \pm 0.8$ & $270.3 \pm 1.1$ & $223.0 \pm 1.2$ & $173.8 \pm 0.6$ & $143.2 \pm 1.2$ & 47.0 \\
\hline
\end{tabular}

Note: *Values are mean \pm Standard Deviation (SD), $p<0.05$ when compared with the values of control

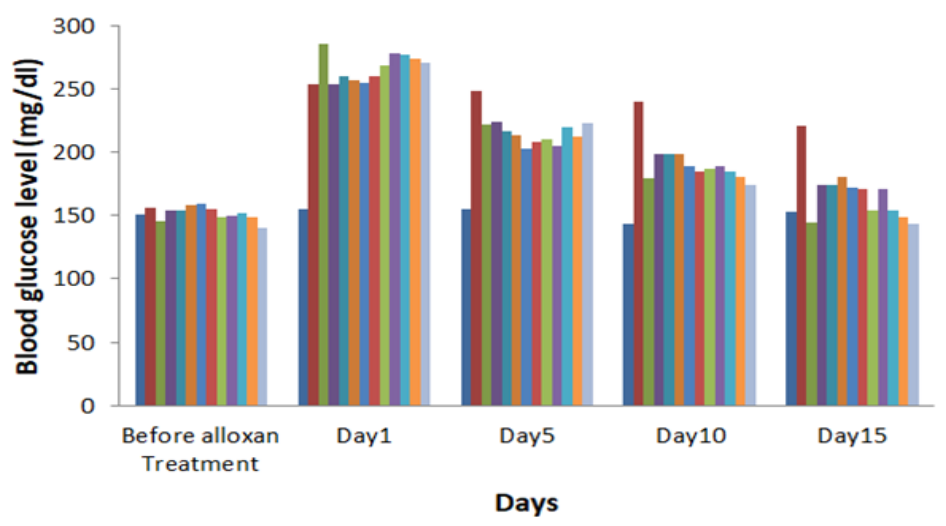

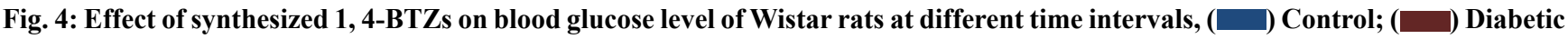
control; $(\square)$ Metformin; $(\square) 3 \mathrm{a} ;(\square) 3 \mathrm{~b} ;(\square) 3 \mathrm{c} ;(\square) 3 \mathrm{~d} ;(\square) 3 \mathrm{e} ;(\square)$ 3f; $(\square) 3 \mathrm{~g} ;(\square) 3 \mathrm{~h} ;(\square) 3 \mathrm{i} ;(\square)$ CGP37157

Based on the information obtained from the previous studies that BTZ nucleus has antidiabetic potential, the present study was aimed to perform the synthesis and evaluation of antihyperglycemic activity of 1,4-BTZs (3a-i). The spectral data confirmed the formation of 3a-i. Results of antihyperglycemic study in alloxaninduced diabetic rats showed that synthesized 1,4-BTZs reduce the elevated blood glucose level to an optimum level. It was revealed that electronegative substitution at 7 and 2' position of 1,4-BTZs was favorable for antihyperglycemic activity. From the above results, it was concluded that this study may be helpful in development of some novel antidiabetic agents.

\section{Acknowledgements:}

Authors are thankful to Director of Shri Govindram Seksaria Institute of Technology and Science, Indore for providing necessary facilities for research, Head of Department, School of Pharmacy, Devi Ahilya
Vishwavidyalaya, Indore for biological evaluation and IPCA Laboratories Ltd. Ratlam, Madhya Pradesh for providing gift sample of metformin.

\section{Conflicts of interest:}

The authors declared no conflict of interest.

\section{REFERENCES}

1. Kenny SJ, Aubert RE, Geiss LS. Prevalence and incidence of non-insulin-dependent diabetes. Diabetes 1995;2:47-67.

2. Buse JB, Wexler DJ, Tsapas A, Rossing P, Mingrone G, Mathieu C, et al. 2019 update to: management of hyperglycemia in type 2 diabetes, 2018. A consensus report by the American diabetes association (ADA) and the European association for the study of diabetes (EASD). Diabetes care 2020;43(2):487-93.

3. Pari L, Amarnath Satheesh M. Antidiabetic effect of Boerhavia diffusa: effect on serum and tissue lipids in experimental diabetes. J Med Food 2004;7(4):472-6.

4. Krentz AJ, Ferner RE, Bailey CJ. Comparative tolerability profiles of oral antidiabetic agents. Drug Saf 1994;11(4):22341. 
5. DeFronzo RA. Pharmacologic therapy for type 2 diabetes mellitus. Ann Intern Med 2000;133(1):73-4.

6. Nuss JM, Wagman AS. Recent advances in therapeutic approaches to type 2 diabetes. Annu Rep Med Chem 2000;35:211-20.

7. Nissen SE, Wolski K. Effect of rosiglitazone on the risk of myocardial infarction and death from cardiovascular causes. $\mathrm{N}$ Engl J Med 2007;356(24):2457-71.

8. Lewis JD, Ferrara A, Peng T, Hedderson M, Bilker WB, Quesenberry CP, et al. Risk of bladder cancer among diabetic patients treated with pioglitazone: interim report of a longitudinal cohort study. Diabetes Care 2011;34(4):916-22.

9. Davis S, Alonso MD. Hypoglycemia as a barrier to glycemic control. J Diabetes Complications 2004;18(1):60-8.

10. Matveyenko AV, Dry S, Cox HI, Moshtaghian A, Gurlo T, Galasso $\mathrm{R}$, et al. Beneficial endocrine but adverse exocrine effects of sitagliptin in the human islet amyloid polypeptide transgenic rat model of type 2 diabetes: interactions with metformin. Diabetes 2009;58(7):1604-15.

11. Butler AE, Campbell-Thompson M, Gurlo T, Dawson DW, Atkinson M, Butler PC. Marked expansion of exocrine and endocrine pancreas with incretin therapy in humans with increased exocrine pancreas dysplasia and the potential for glucagon-producing neuroendocrine tumors. Diabetes 2013;62(7):2595-604.

12. Egan AG, Blind E, Dunder K, de Graeff PA, Hummer BT, Bourcier T, et al. Pancreatic safety of incretin-based drugsFDA and EMA assessment. N Engl J Med 2014;370(9):794-7.

13. Pei Y, Lilly MJ, Owen DJ, D'Souza LJ, Tang XQ, Yu J, et al. Efficient syntheses of benzothiazepines as antagonists for the mitochondrial sodium-calcium exchanger: potential therapeutics for type II diabetes. J Org Chem 2003;68(1):92103.

14. Cox DA, Matlib MA. Modulation of intramitochondrial free $\mathrm{Ca}^{2+}$ concentration by antagonists of $\mathrm{Na}^{+}-\mathrm{Ca}^{2+}$ exchange. Trends Pharmacol Sci 1993;14(11):408-13.

15. Siegel EG, Wollheim CB, Renold AE, Sharp GW. Evidence for the involvement of $\mathrm{Na} / \mathrm{Ca}$ exchange in glucose-induced insulin release from rat pancreatic islets. J Clin Invest 1980;66(5):9961003.

16. Van Eylen F, Antoine MH, Lebrun P, Herchuelz A. Inhibition of $\mathrm{Na} / \mathrm{Ca}$ exchange stimulates insulin release from isolated rat pancreatic islets. Fundam Clin Pharmacol 1994;8(5):425-9.

17. Lee B, Miles PD, Vargas L, Luan P, Glasco S, Kushnareva $\mathrm{Y}$, et al. Inhibition of mitochondrial $\mathrm{Na}^{+}-\mathrm{Ca}^{2+}$ exchanger increases mitochondrial metabolism and potentiates glucosestimulated insulin secretion in rat pancreatic islets. Diabetes 2003;52(4):965-73.

18. Anderson C, Davis R, Ghosh S, Pei Y. Treatment of diabetes mellitus by inhibition of mitochondrial calcium/sodium antiporter. Mitokor US 20020082193A1 2002:1-23.

19. Dasoondi AS, Singh V, Voleti SR, Tiwari M. Comparative molecular field analysis of benzothiazepine derivatives: Mitochondrial sodium calcium exchange inhibitors as antidiabetic agents. Indian J Pharm Sci 2008;70(2):186.

20. Tsaioun K, Kates SA. De-risking drug discovery programmes early with ADMET. In: Kapetanovic IM, ed. Drug discovery and development-Present and future: InTech, Janeza, Croatia; 2011. p. 275-94.

21. Sternbach LH, Fryer RI, Metlesics W, Sach G, Stempel A. Quinazolines and 1, 4-benzodiazepines. V. o-aminobenzophenones1a, b. J Org Chem 1962;27(11):3781-8.

22. Lipinski CA, Lombardo F, Dominy BW, Feeney PJ.
Experimental and computational approaches to estimate solubility and permeability in drug discovery and development settings. Adv Drug Deliv Rev 1997;23(1-3):3-25.

23. Congreve M, Carr R, Murray C, Jhoti H. A rule of three for fragment-based lead discovery?. Drug Discov Today 2003;8(19):876-7.

24. Ghose AK, Viswanadhan VN, Wendoloski JJ. A knowledgebased approach in designing combinatorial or medicinal chemistry libraries for drug discovery. 1. A qualitative and quantitative characterization of known drug databases. J Comb Chem 1999;1(1):55-68.

25. QikProp 3.3. Rapid ADME predictions of drug candidates. Schrodinger-Suite LLC: New York; 2010.

26. Ioakimidis L, Thoukydidis L, Mirza A, Naeem S, Reynisson J. Benchmarking the reliability of QikProp. Correlation between experimental and predicted values. QSAR Comb Sci 2008;27(4):445-56.

27. Srivastav VK, Tiwari M, Zhang X, Yao XJ. Synthesis and antiretroviral activity of 6-acetyl-coumarin derivatives against HIV-1 infection. Indian J Pharm Sci 2018;80(1):108-17.

28. Ntie-Kang F. An in silico evaluation of the ADMET profile of the StreptomeDB database. Springerplus 2013;2(1):1-1.

29. Sander T, Freyss J, von Korff M, Rufener C. DataWarrior: an open-source program for chemistry aware data visualization and analysis. J Chem Inf Model 2015;55(2):460-73.

30. Srivastav VK, Tiwari M. k-nearest neighbor molecular field analysis based 3D-QSAR and in silico ADME/T studies of cinnamoyl derivatives as HIV-1 integrase inhibitors. Med Chem Res 2015;24(2):684-700.

31. Sander T. OSIRIS property explorer. Organic Chemistry Portal; 2001.

32. Verma L, Khatri A, Kaushik B, Patil UK, Pawar RS. Antidiabetic activity of Cassia occidentalis (Linn) in normal and alloxaninduced diabetic rats. Indian J Pharmacol 2010;42(4):224.

33. Sachan N, Thareja S, Agarwal R, Kadam SS, Kulkarni VM. Substituted biphenyl ethanones as antidiabetic agents: synthesis and in-vivo screening. Int J Pharm Tech Res 2009;1:625-31.

34. Tenpe CR, Yeole PG. Comparative evaluation of antidiabetic activity of some marketed polyherbal formulations in alloxan induced diabetic rats. Int J Pharm Tech Res 2009;1(1):43-9.

35. Mariappan G, Saha BP, Datta S, Kumar D, Haldar PK. Design, synthesis and antidiabetic evaluation of oxazolone derivatives. J Chem Sci 2011;123(3):335-41.

36. Litchfield JJ, Wilcoxon FR. A simplified method of evaluating dose-effect experiments. J Pharmacol Exp Ther 1949;96(2):99113.

37. Perfumi M, Tacconi R. Antihyperglycemic effect of fresh Opuntia dillenii fruit from Tenerife (Canary Islands). Int J Pharmacogn 1996;34(1):41-7.

38. Waisundara VY, Hsu A, Huang D, Tan BK. Scutellaria baicalensis enhances the anti-diabetic activity of metformin in streptozotocin-induced diabetic Wistar rats. Am J Chin Med 2008;36(03):517-40.

39. Pattan SR, Suresh CH, Pujar VD, Reddy VV, Rasal VP, Koti BC. Synthesis and antidiabetic activity of 2-amino [5'(4-sulphonylbenzylidine)-2,4-thiazolidinedione]-7chloro-6-fluorobenzothiazole. Indian J Chem Sect B 2005; 44B:2404-8.

40. Carey F. Reduction of ketones: Preparation of benzhydrol. $7^{\text {th }}$ ed. Organic Chemistry. McGraw-Hill 2008;15:622-8.

41. Pavia DL, Lampman GM, Kriz GS. Introduction to spectroscopy: A Guide for students of organic chemistry. $3^{\text {rd }}$ ed. USA: Thomson Learning, Inc.; 2001:29-82. 